\title{
Iranian Society of Cardiac Surgeons Position Statement for the Treatment of Patients in Need of Cardiac Surgery in the COVID-19 Pandemic Period (Version I)
}

Esmaeil Asdaghpour ${ }^{1}$, Ramin Baghaei ${ }^{2}$, Naser Jalilifar ${ }^{3}$, Hassan Radmehr ${ }^{4}$, Mahmoud Shirzad ${ }^{5}$, Mohammad Reza Mirzaaghayan ${ }^{4}$, Mohhamad Ali Yousefnia ${ }^{6}$, Nicholas Austin ${ }^{7}$, Alireza Alizadeh Ghavidel (1D ${ }^{8,}{ }^{*}$, Zargham Hossein Ahmadi ${ }^{9}$, Ahmad Ali Amirghofran ${ }^{10}$, Ahmad Amin ${ }^{11}$, Kamran Babazadeh ${ }^{12}$, Rezayat Parvizi ${ }^{13}$, Saeid Hosseini ${ }^{14}$, Amir Nasser Jadbabaei ${ }^{9}$, Alireza Jahangirifard ${ }^{15}$, Manouchehr Hekmat ${ }^{2}$, Minoosh Shabani ${ }^{16}$, Parham Sadeghipour ${ }^{17}$, Mohammad Abbasi Tashnizee ${ }^{18}$, Maziar Gholampour Dehaki ${ }^{8}$, Ata Firouzi ${ }^{17}$, Mohsen Mirmohammadsadeghi ${ }^{19}$ and Akbar Nikpajouh ${ }^{20}$

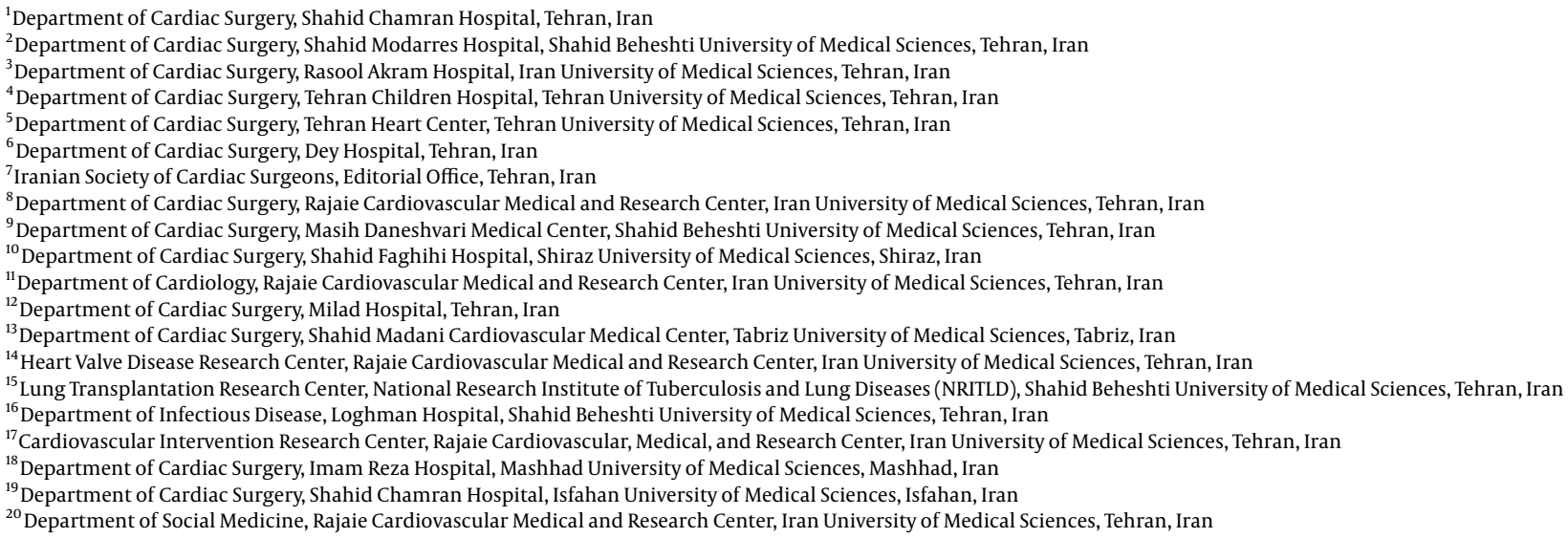

"Corresponding author: Professor of Cardiac Surgery, Heart Valve Disease Research Center, Rajaie Cardiovascular Medical and Research Center, Iran University of Medical Sciences, Tehran, Iran. Email: aaghavidel@gmail.com

Received 2020 April 28; Revised 2020 May 11; Accepted 2020 May 11.

\begin{abstract}
The effects of recent Covid-19 pandemic on this planet must be viewed with a wise eye and we should learn that human beings are interconnected chains, and that ignoring the laws of existence will undoubtedly continue with reflections similar to the way we are today. Although the community of heart surgeons is not at the forefront of the treatment of this epidemic, they are ready to rush to the aid of other colleagues if necessary. The aim of preparing this protocol is to prioritize cardiac surgery procedures, maintain blood and blood product reserves and provide the appropriate care for patients while taking precautions for the safety of medical staff. The general recommendation in this first version of protocol is to postpone all elective cardiac surgeries and perform emergent and urgent cases according to suggested personal protection strategies for Covid-19.
\end{abstract}

Keywords: Cardiac Surgery, Outbreak, Protocol, Pandemic, Covid-19, PPE, SARS-Cov-2

\section{Context}

The effects of recent Covid-19 pandemic on this planet must be viewed with a wise eye and we should learn that human beings are interconnected chains, and that ignor- ing the laws of existence will undoubtedly continue with reflections similar to the way we are today. Our country is one of the first countries involved after China (1). In recent weeks, the number of deaths and injuries have in- 
creased. Fortunately, the country's health team has done its heavy, indescribable and excessive responsibility well and deserves a lot of praise, despite all the restrictions and sanctions. Although the community of heart surgeons is not at the forefront of the treatment of this epidemic, they are ready to rush to the aid of other colleagues if necessary. While appreciating the teams involved, the Iranian Society of Cardiac Surgeons (ISCS) considers its responsibility to provide a clear and scientific framework for co-operative heart surgeons during and after the Coronavirus Crisis in order to indirectly assist the team.

For optimal use of ICU beds, medical teams, blood and blood products, and personal protective equipment (PPE) it is required to change the usual approach. We hope by using the new approach, while continuing to provide quality service for heart patients, make the best use of available resources. While appreciating the support of the esteemed members of ISCS in observing the provisions of this protocol, it is expected that the esteemed officials of the Ministry of Health and Medical Education facilitate the implementation of the provisions of this protocol with the necessary assistance.

\section{Priorities for Cardiac Surgery in Covid-19 Epidemic Status}

The classification of cardiac surgery in recent pandemic status is based on the latest world-renowned guidelines adopted by the Heart Team. In pandemic situations, the use of non-surgical approaches is preferred if similar results can be obtained (2). To facilitate the decisionmaking process, patients are divided into three categories of Urgent, Emergent, and Elective (3).

All Emergent and Urgent actions in this epidemic must be carried out in compliance with safety precautions, although this has been the case in recent weeks. Until having effective control of this epidemic and permission from the Ministry of Health, it is necessary to postpone all elective cardiac surgeries.

\subsection{Cardiac Surgery in Covid-19 Patients}

In patients with Covid-19 who require emergency or urgent cardiac surgery, the decision is made based on the prognosis of the current and underlying disease, life expectancy, and the recommendation of the COVID team consisting of a cardiovascular surgeon, a cardiologist, cardiac anesthesia fellowship, intensive care specialist, infectious disease specialist and pulmonologist. The risk of medical team involvement is high during the surgery process of patients with Covid-19 infection, so the general recommendation is that these procedures be limited for salvage cardiac operations during acute phase of infection and other emergent and urgent surgeries should be postponed until the patient has relatively recovered from covid-19 infection and become clinically stable (3). Since the probability of mortality and morbidity of heart surgery in Covid-19 patients is significantly higher (at least 21\%) this delay, if possible, is also in patient's favor until the patient recovers (4-7).

\subsection{Cardiac Surgery in Non-Covid-19 Patients}

The general recommendation is that these patients should not be operated in referral centers for Covid-19 as much as possible, and referred to the hospitals that are not involved with Covid-19.

A. Emergent surgery: Surgeries that need to be performed within the first few hours after diagnosis; for example, acute type A dissection, patients with cardiac trauma, mechanical valve thrombosis and CAD with ongoing ischemia that have not responded to non-surgical treatment. (Table 1)

B. Urgent surgery: Surgeries that need to be performed in the next few days in the same hospitalization setting after the diagnosis; such as patients with severe CAD with low threshold angina without non-surgical options or patients with heart failure secondary to structural heart disease who are temporarily controlled with medical treatment but needs surgical treatment in next step (Table 1)

C. Elective surgery: Surgeries that could be postponed for a couple of weeks in patients without life threating diseases, such as chronic MR, ASD and any stable or controlled CAD by non-surgical methods.

\section{Personal Protection in the Surgical Team}

A. Private office and clinic: The necessary instructions in these cases have been issued by the deputy of the Ministry of Health and Medical Education and Iranian Medical Council Letters No. 369/11/111/399 dated 6 April 2020 found in attachments number 1 and 2.

B. Hospital and operating room: In this case, patients are divided into low-risk and high-risk groups in terms of Covid-19 involvement. In the high-risk group, if surgery cannot be postponed due to patient's clinical conditions, it is recommended that the surgery be performed in the operating rooms assigned for Covid-19 patients and then be transferred to the ICU-OH, specified for Covid-19 patients if available.

Patients in the low-risk group can be operated in standard operating room and ICUs.

In the Figure 1, the decision-making process and the necessary actions are summarized. 


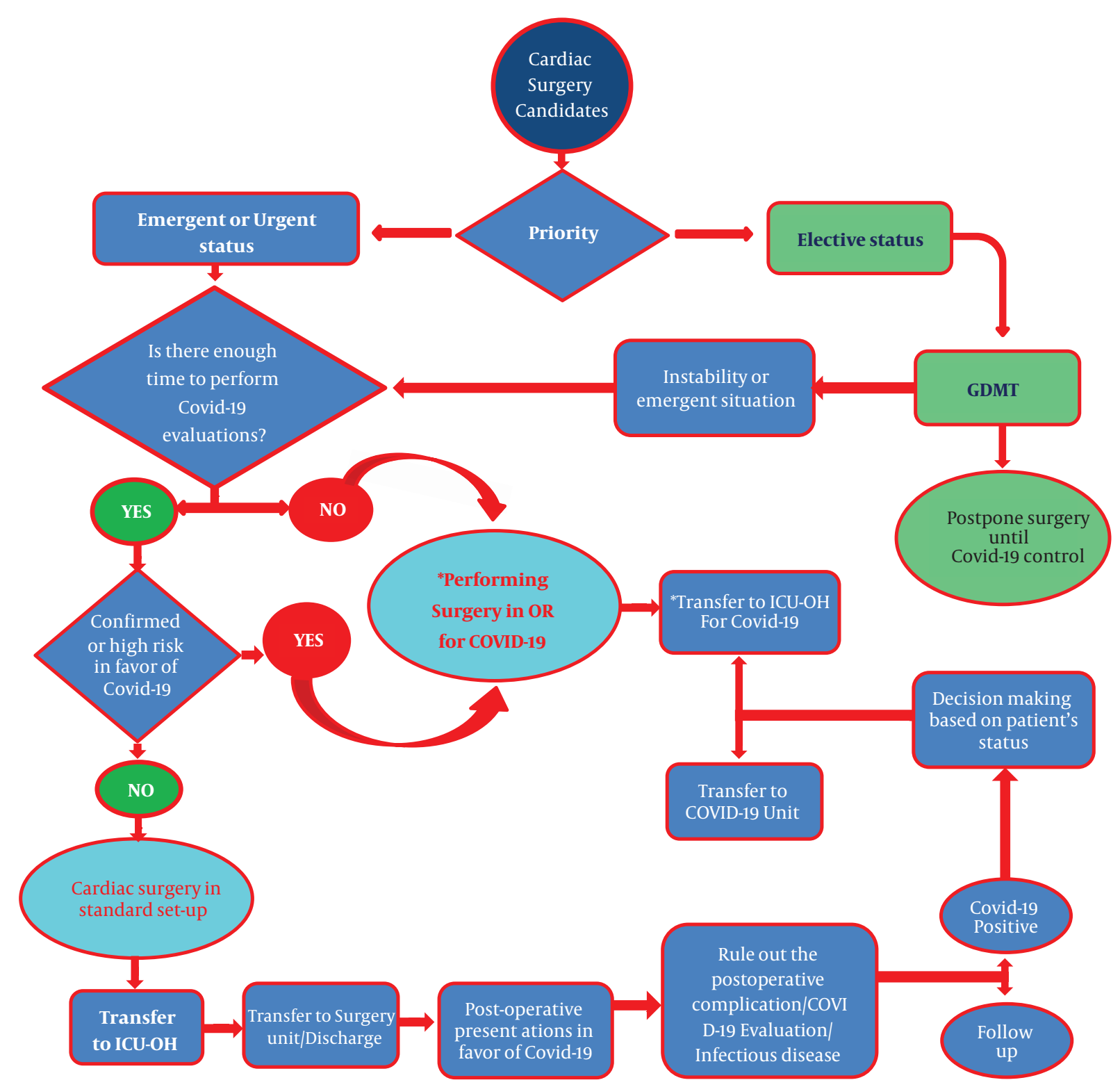

Figure 1. Algorithm for the management of cardiac surgery patients during Covid-19 outbreak (GDMT: Guideline directed medical therapy. ${ }^{*}$ All medical staff entering the OR must wear N95 mask (be fit-tested) in addition to standard precaution.)

\section{High Risk Patients}

A. Patients with definite Covid-19 infection, based on Covid-19 laboratory tests, CT scan and clinical symptoms.

B. Patients who have had a Covid-19 test because of suspected clinical symptoms and are waiting for the result.

C. Patients who have had unprotected contact with positive Covid-19 cases in the past two weeks.

D. Suspected patients who need emergent operation and there is not enough time for evaluation with diagnostic tools like Covid-19 test and chest CT scan (8).

\section{Low Risk Patients}

A. Lack of paraclinical findings in favor of recent Covid19 involvement, including CT scans or specific Covid-19 tests or non-specific tests such as (CBC, CRP, LDH) 


\begin{tabular}{|c|c|c|}
\hline No. & Emergent Operations & Urgent Operations \\
\hline 1 & Cardiac and great artery trauma & $\begin{array}{l}\text { CAD patient with low threshold } \\
\text { angina (Class III-IV) }\end{array}$ \\
\hline 2 & Acute type A dissection & $\begin{array}{l}\text { Patients with severe } \\
\text { symptomatic AS }\end{array}$ \\
\hline 3 & Cardiac tamponade & $\begin{array}{l}\text { NVE or PVE after optimal } \\
\text { Antibiotic and medical therapy } \\
\text { or complicated endocarditis }\end{array}$ \\
\hline 4 & Mechanical valve thrombosis & Acute MR(Flail MV) \\
\hline 5 & $\begin{array}{l}\text { Acute pulmonary emboli (with } \\
\text { no medical or interventional } \\
\text { options) }\end{array}$ & $\begin{array}{l}\text { Post-MI complications (VSD, LV } \\
\text { false aneurysm, sever acute MR) }\end{array}$ \\
\hline 6 & $\begin{array}{l}\text { Patients with ACS and sever CAD } \\
\text { (Significant LM lesions or high } \\
\text { SYNTAX score) }\end{array}$ & Post-operative mediastinitis \\
\hline 7 & $\begin{array}{l}\text { Post PCI or other percutaneous } \\
\text { approaches sever complications }\end{array}$ & $\begin{array}{l}\text { CAD patients with ostial LAD } \\
\text { lesion amenable to } \\
\text { percutaneous management }\end{array}$ \\
\hline 8 & $\begin{array}{l}\text { Ongoing ischemia in CAD } \\
\text { Patients uncontrolled by } \\
\text { percutaneous approaches and } \\
\text { GDMT }\end{array}$ & $\begin{array}{l}\text { Large right heart clots }(>20 \\
\text { mm) with no other non-surgical } \\
\text { treatment option }\end{array}$ \\
\hline 9 & $\begin{array}{l}\text { Patients with STEMI not suitable } \\
\text { response to GDMT or } \\
\text { percutaneous approaches (or } \\
\text { have ongoing ischemia after } \\
\text { culprit lesion PCI) }\end{array}$ & $\begin{array}{l}\text { Large hypermobile left heart } \\
\text { clots }(>10 \mathrm{~mm}) \text { with no other } \\
\text { non-surgical treatment option }\end{array}$ \\
\hline 10 & CHD patients with cyanotic spell & LA Myxoma \\
\hline 11 & $\begin{array}{l}\text { Prostaglandin dependent CHD } \\
\text { patients }\end{array}$ & $\begin{array}{l}\text { Ascending Aorta aneurysm } \\
\text { larger than } 60 \mathrm{~mm}\end{array}$ \\
\hline 12 & $\begin{array}{l}\text { Individualized cases (according } \\
\text { to the heart team decision } \\
\text { making) }\end{array}$ & $\begin{array}{l}\text { Subacute and chronic type A } \\
\text { dissection }\end{array}$ \\
\hline 13 & & $\begin{array}{l}\text { TGA, Truncus arteriosus, A-P } \\
\text { window, TAPVC, Aorta } \\
\text { interruption }\end{array}$ \\
\hline 14 & & $\begin{array}{l}\text { CHD with L-R shunt, pulmonary } \\
\text { artery hypertension in critical } \\
\text { age groups }\end{array}$ \\
\hline 15 & & $\begin{array}{l}\text { Individualized cases (according } \\
\text { to the heart team decision } \\
\text { making) }\end{array}$ \\
\hline
\end{tabular}

Abbreviations: A-P window, Aortopulmonary window; ACS, Acute coronary syndrome; AS, Aortic stenosis; CAD, Coronary artery disease; $\mathrm{CHD}$, Congenital heart disease; GDMT, Guideline directed medical therapy; LA, Left atrium; LM, Left main; LV, Left ventricle; MI, Myocardial infraction; MR, Mitral regurgitation; MV, Mitral valve; NVE, Native valve endocarditis, PCI, Percutaneous coronary intervention; PVE, Prosthetic valve endocarditis, STEMI, S-T elevation myocardial infraction; TAPVC, Total anomalus of pulmonary vein connection; TGA, Transposition of great arteries; VSD, Ventricular septal defect

B. Lack of clinical findings in favor of Covid-19 in the last two weeks

In the low-risk group, standard surgical care for both patients and surgical team. However, in Coronavirus epidemic, more precise protective care including frequent hand washing, use of hand sanitizers, surgical masks and gloves are recommended.

Recommendations for protection of the medical staff during the surgery of high-risk patients:

1. Using Respirator, or double mask(N95 mask and surgical mask), goggles, gloves and full body disposable coverall and strict observance of the principles of using PPE.

2. Using two surgical gloves on top of each other

3. Using surgical suction to discharge gases emitted from electrocautery

4. Use the operating room allocated for Covid-19 if possible

5. Using the operating room equipped with negative pressure if possible

6. Minimal presence of surgical team in the operating room, especially during induction of anesthesia and CPR (9).

\section{How to Inform the Patient and Their Family during Covid-19 Epidemic}

Due to the rightful concerns of patients and their families at this time, it is necessary for the surgeon to provide sufficient information in this regard and obtain the informed consent. Highlights are as follows:

1. Justifying the patient and her/his family about the reason of emergent or urgent cardiac surgery during Covid-19 pandemic.

2. Obtaining written consent and sufficient explanation of the possibility of Covid-19 infection after surgery or conversion of the disease from asymptomatic to symptomatic form, increased risk of disease due to frequent contact with the medical staff, temporary weakened immune system deficiency due to the use of cardiopulmonary bypass (CPB), blood and blood products transfusions

3. Clarifying to the patients and their families that there is not enough evidence that cardiac surgery increases the risk of Covid-19 disease independently, however, if the patients involve with this virus in perioperative period, they will experience a more severe infection and serious complications.

4. Explaining that heart surgery in a Covid-19 patient (even after an acute phase) may increase the risk of mortality and morbidities.

5. Explaining to patients and their families about the increased risk of death from Covid-19 involvement during perioperative period for patients with underlying diseases such as chronic lung disease (COPD), diabetes, immune system deficiency for any reason, old age and heart failure.

6 . Justifying the patients and their families about the restrictions of regular visits of patient according to the directive of the Ministry of Health and Medical Education. 
6.1. Are There Any Restrictions on Transfusion of Blood and Blood Products During the Surgery at This Period of Time?

There are not enough studies, but so far there have been no reports of transmission of the disease through blood and blood products or contact with an infected person's blood (10). Therefore, transfusion of blood and blood products is not a problem in cardiac surgery, but the general recommendation is to perform meticulous surgery and follow the principles of Patient Blood Management to minimize the need for blood and blood products transfusion in order to reduce the patients' complications and also maintain blood bank reserves (11).

\subsection{Is Covid-19 Transmitted Through Blood? (Patient's Blood to the Medical Staff and Vice Versa)}

There are not enough studies, however, there is still no report of the transmission of the disease via patients' blood and body secretions such as urine and feces (12). There is still no screening test for Covid-19 during blood donation so the medical staff should be careful and cautious about unprotected blood products contact, although the virus is mainly concentrated in the mouth, tongue and respiratory tract.

According to existing protocols from blood transfusion organizations, blood donations are not made from donors who have had suspected symptoms of the disease in the last 28 days.

\section{The Role of ECMO in Patients with Covid-19}

Not many studies have been published in this regard, although successful results by ECMO had been reported for management of ARDS during previous SARS epidemics (13). Also there are reports of the usefulness of ECMO in the treatment of severe cases of Covid-19 disease (14). However, the results of different countries do not match well, and the major benefits of ECMO have been reported from China and Europe, especially France. There is no published study from our country, but the experiences of our colleagues at Masih Daneshvari Hospital are valuable.

At present, it is not possible to determine the specific criteria for using ECMO with the available information, but what is clear is that ECMO should be used in specific patients by a trained team in a well-equipped center with sufficient ECMO experience for non Covid-19 patients. Recently, the colleagues of Rajaie Cardiovascular, Medical and Research Center and Masih Daneshvari Hospital are preparing a good protocol in this regard that can be used.
7.1. Cardiopulmonary Resuscitation in Cardiac Surgery Patients During Covid-19 Pandemics

A good protocol in this regard has been prepared by the Iranian Heart Association, which can be used (15).

Heart failure surgery including heart transplant and ventricular assist device (LVAD) implantation:

Due to the high vulnerability of these patients and the predisposing conditions for Covid-19 in these surgeries, it is recommended that these surgeries be performed only for critically ill patients (Intermacs 1,2) and be carried out in accordance with the approvals of the Committee for the Provision and Organ Transplantation of the Ministry of Health and Medical Education.

7.2. Management of Patients who Experience Suspicious Symptoms of Covid-19 After Heart Surgery?

The treatment of these patients is based on the latest national protocols and good experiences of colleagues in different medical centers. However, since the mortality rate from coronavirus infection after surgery is over $21 \%$ and the need for ICU is over $41 \%$, in case of any clinical symptoms suspected of Covid-19 disease during recovery period (such as fever, shortness of breath, excessive fatigue) besides early hospitalization and outside the usual Covid-19 protocols and rule out of early post-op complications, the patient's clinical condition should be carefully monitored and the possibility of corona involvement should be carefully assessed.

Outpatient stable cases with suspicious clinical symptoms such as mild shortness of breath, cough, fever and myalgia, performing routine diagnostic tests including cell count, LDH check, CRP, Covid-19 test, and chest CT scan is recommended. It is also recommended that patients undergo transthoracic echocardiography (TTE) to evaluate post-op problems such as pericardial effusion, heart failure, valvular insufficiency, and so on that may mimic Covid-19 symptoms. It is recommended that patients suspected of having Covid-19 infection be referred to specific hospitals as soon as possible after the complications of the operation have been ruled out.

CME and other educational programs of ISCS in Pandemics and post Pandemics status:

A. The bi-monthly ISCS meetings will be held virtually using "Zoom Meeting" and other "Group Chatting" soft wares, until the complete elimination of the disease and the permission of the national Covid-19 headquarters.

B. Other regional or national scientific conferences shall be adjourned until further notice and with the permission of the national Covid-19 headquarters. 


\subsection{Patient Follow-Up After Discharge}

It is recommended that telephone calls and the optimal use of telemedicine be replaced by the usual way of visiting patients.

\section{Footnotes}

Authors' Contribution: Dr. Yousefnia, Dr. Shirzad, Dr. Asdaghpour, and Dr. Jalilifar: study design. Dr. Radmehr and Dr. Baghaie: critical revision of the manuscript for important intellectual content. Dr. Alizadeh Ghavidel, Dr. Austine, and Dr. Mirzaaghayan: study design, concept and drafting the manuscript. Dr. Hossein Ahmadi, Dr. Amirghofran, Dr. Amin, Dr. Babazadeh, Dr. Parvizi, Dr. Hosseini, Dr. Jadbabaei, Dr. Jahangiri Fard, Dr. Hekmat, Dr. Shabani, Dr. Sadeghipour, Dr. Abbasi, Dr. Gholampour Dahaki, Dr. Firouzi, Dr. Mirmohammadsadeghi, and Dr. Nikpajouh: review the protocol.

Conflict of Interests: The authors and reviewers of this manuscript declare no conflicts of interest.

Funding/Support: There was no financial support provided for this protocol.

\section{References}

1. Ghaffarzadegan N, Rahmandad H. Simulation-based Estimation of the Spread of COVID-19 in Iran. medRxiv. 2020. doi: 10.1101/2020.03.22.20040956.

2. Iranian Health Ministry. National flowchart of dignosis and treatment of COVID-19 in outpatient and inpatient settings 2020. [updated 25 March 2020]. Available from: https://irimc.org/Portals/0/NewsAttachment.

3. Stahel PF. How to risk-stratify elective surgery during the COVID-19 pandemic? Patient Saf Surg. 2020;14:8. doi: 10.1186/s13037-020-002359. [PubMed: 32288785]. [PubMed Central: PMC7107008].

4. Lei S, Jiang F, Su W, Chen C, Chen J, Mei W, et al. Clinical characteristics and outcomes of patients undergoing surgeries during the incubation period of COVID-19 infection. EClinicalMedicine. 2020:100331.
5. Clerkin KJ, Fried JA, Raikhelkar J, Sayer G, Griffin JM, Masoumi A, et al. Coronavirus Disease 2019 (COVID-19) and Cardiovascular Disease. Circulation. 2020. doi:10.1161/CIRCULATIONAHA.120.046941. [PubMed: 32200663].

6. Xiong TY, Redwood S, Prendergast B, Chen M. Coronaviruses and the cardiovascular system: acute and long-term implications. Eur Heart J. 2020;41(19):1798-800. doi: 10.1093/eurheartj/ehaa231. [PubMed 32186331].

7. Li YK, Peng S, Li LQ, Wang Q, Ping W, Zhang N, et al. Clinical and Transmission Characteristics of Covid-19 - A Retrospective Study of 25 Cases from a Single Thoracic Surgery Department. Curr Med Sci. 2020;40(2):295-300. doi: 10.1007/s11596-020-2176-2. [PubMed: 32232652]. [PubMed Central: PMC7104422].

8. He Y, Wei J, Bian J, Guo K, Lu J, Mei W, et al. Chinese Society of Anesthesiology expert consensus on anesthetic management of cardiac surgical patients with suspected or confirmed coronavirus disease 2019. Journal of Cardiothoracic and Vascular Anesthesia. 2020.

9. Ti LK, Ang LS, Foong TW, Ng BSW. What we do when a COVID-19 patient needs an operation: operating room preparation and guidance. Can JAnaesth. 2020;67(6):756-8. doi: 10.1007/s12630-020-01617-4. [PubMed 32144591]. [PubMed Central: PMC7090746].

10. Kwon SY, Kim EJ, Jung YS, Jang JS, Cho NS. Post-donation COVID-19 identification in blood donors. Vox Sang. 2020. doi: 10.1111/vox.12925. [PubMed: 32240537].

11. Mohammadi S, Tabatabaei Yazdi SM, Eshghi P, Norooznezhad AH Coronavirus disease 2019 (COVID-19) and decrease in blood donation: experience of Iranian Blood Transfusion Organization (IBTO). Vox Sang. 2020. doi: 10.1111/vox.12930. [PubMed: 32270880].

12. Interim US. Guidance for Risk Assessment and Public Health Management of Healthcare Personnel with Potential Exposure in a Healthcare Setting to Patients with Coronavirus Disease (COVID-19). 2020. Available from: https://www.cdc.gov/coronavirus/2019-ncov/hcp/guidancerisk-assesmen.

13. Maves RC, Jamros CM, Smith AG. Intensive Care Unit Preparedness During Pandemics and Other Biological Threats. Crit Care Clin. 2019;35(4):609-18. doi: 10.1016/j.ccc.2019.06.001. [PubMed: 31445608]. [PubMed Central: PMC7134984].

14. Ramanathan K, Antognini D, Combes A, Paden M, Zakhary B, Ogino M et al. Planning and provision of ECMO services for severe ARDS during the COVID-19 pandemic and other outbreaks of emerging infectious diseases. Lancet Respir Med. 2020;8(5):518-26. doi: 10.1016/S22132600(20)30121-1. [PubMed: 32203711]. [PubMed Central: PMC7102637].

15. Baay M, Garjani K, Barati S, Payandemehr P, Totonchi Z, Zanganehfar $\mathrm{M}$, et al. Iranian heart association task force on cardiopulmonary resuscitation guidelines on the COVID-19 outbreak. Research in Cardiovascular Medicine. 2020;9(1):3. doi:10.4103/rcm.rcm _10_20. 